\title{
Role of nutrition in liver transplantation for end-stage chronic liver disease
}

\author{
Felix Stickel, Daniel Inderbitzin, and Daniel Candinas
}

\begin{abstract}
Patients with end-stage liver disease often reveal significant protein-energy malnutrition, which may deteriorate after listing for transplantation. Since malnutrition affects post-transplant survival, precise assessment must be an integral part of pre-and post-surgical management. While there is wide agreement that aggressive treatment of nutritional deficiencies is required, strong scientific evidence supporting nutritional therapy is sparse. In practice, oral nutritional supplements are preferred over parenteral nutrition, but enteral tube feeding may be necessary to maintain adequate calorie intake. Protein restriction should be avoided and administration of branched-chain amino acids may help yield a sufficient protein supply. Specific problems such as micronutrient deficiency, fluid balance, cholestasis, encephalopathy, and comorbid conditions need attention in order to optimize patient outcome.

๑) 2008 International Life Sciences Institute
\end{abstract}

\section{INTRODUCTION}

Orthotopic liver transplantation (OLT) has greatly improved the prognosis of patients with chronic liver failure, and clinical features of declining liver function largely normalize following successful organ replacement. Among the most prevalent complications of chronic liver failure is a marked impairment of the nutritional status due to both primary and secondary malnutrition. The degree of malnutrition has been a parameter of the old version of the Child-Pugh index; however, the lack of universally applicable diagnostic tools to precisely diagnose malnutrition in clinical practice has left the diagnosis based on clinical signs of encephalopathy and ascites as well as the laboratory parameters serum bilirubin, serum albumin, and prothrombin time (index). ${ }^{1}$ Interestingly, the model for end-stage liver disease (MELD), initially developed for prediction of survival of patients with complications of portal hypertension scheduled for a transjugular intrahepatic portosystemic shunt, is now widely used for organ allocation in liver transplant programmes, but it does not consider nutritional status at all. ${ }^{2}$ Possibly, its failure to accurately predict survival in approximately $15-20 \%$ of potential transplant recipients relates to the fact that malnutrition does not influence MELD figures.

No controversy currently exists regarding the importance of nutritional status as an important predictor of post-transplant outcome and the benefits of its therapeutic improvement, although evidence from randomized clinical trials is limited. The present review aims to summarize the current evidence on nutritional aspects in liver transplantation both in the pre- and post-transplant setting in order to highlight the importance of sufficient nutritional support as a valuable intervention to improve patients' overall prognosis and quality of life.

\section{PREVALENCE AND SIGNIFICANCE OF MALNUTRITION IN END-STAGE LIVER DISEASE}

With the exception of patients with fulminant hepatic failure, most candidates for OLT present with significant malnutrition, and nutritional deficiencies usually evolve prior to clinical signs of hepatic insufficiency. Protein

Affiliations: F Stickel is with the Institute of Clinical Pharmacology, Inselspital, University of Berne, Berne, Switzerland. D Inderbitzin and $D$ Candinas are with the Department of Visceral and Transplant Surgery, Inselspital, University of Berne, Berne, Switzerland.

Correspondence: F Stickel, Institute of Clinical Pharmacology, University of Berne, Murtenstrasse 35, 3010 Berne, Switzerland. E-mail: felix.stickel@ikp.unibe.ch, Phone: +41-31-632 8715, Fax: +41-31-632 4997

Key words: branched-chain amino acids, liver transplantation, nutritional therapy, protein malnutrition 
energy malnutrition (PEM), in particular, is frequently encountered in patients with cirrhosis of nearly every etiology. ${ }^{3}$ Even in stable cirrhotic patients, who are commonly referred to as having Child A cirrhosis, protein depletion is prevalent in approximately $20 \%$ of patients. ${ }^{4}$ This figure rises sharply as liver insufficiency progresses, and a majority of patients with Child $\mathrm{C}$ cirrhosis have significant nutritional deficiencies. ${ }^{5-8}$ PEM clinically presents with weakness, muscle wasting, weight loss, nausea, and anorexia; its prevalence is similar in advanced alcoholic liver disease and other causes of liver cirrhosis. ${ }^{7,9}$ In alcoholic cirrhosis, PEM is closely associated with complications of cirrhosis including infections, encephalopathy, development of ascites, and variceal bleeding, ${ }^{10,11}$ as well as with reduced patient and graft survival after OLT. ${ }^{12,13}$

Patients with end-stage liver disease are often deficient in various vitamins and other micronutrients. ${ }^{14}$ Cirrhotic alcoholics are especially susceptible to severe vitamin depletion, particularly that of folate and pyridoxal-5' -phosphate, the biologically active coenzyme of vitamin B6, with both occurring in up to $70 \%$ of cases. ${ }^{15}$ Thiamine levels are also frequently decreased in patients with alcoholic and hepatitis C-related cirrhosis, which may elicit the Wernicke-Korsakoff syndrome and BeriBeri cardiomyopathy. ${ }^{16}$ A typical feature of early and advanced alcoholic liver disease is an increasingly severe reduction of hepatic vitamin A stores, which sometimes leads to infertility and night blindness. ${ }^{17}$ In vitamin A-deficient cirrhotics, its supplementation, even at relatively moderate doses, may further aggravate liver injury since high-dose vitamin A preparations may be hepatotoxic due to polar retinoid metabolites that cause hepatocellular apoptosis and may promote fibrogenesis. ${ }^{18,19}$ Zinc deficiency is common in patients with decompensated cirrhosis and likely relates to decreased absorption and a diuretics-induced increase in its urinary excretion. Clinically, zinc deficiency presents with alterations of smell and taste, protein metabolism, and encephalopathy. Regarding the latter, one study showed that zinc supplementation resulted in lower ammonia levels following an alanine challenge, and improvements of psychometric tests, liver function, and Child-Pugh score,$^{20}$ but another study did not replicate these findings. ${ }^{21}$

Reduced nutritional status has been identified as an independent predictor of poor prognosis in patients with liver cirrhosis ${ }^{22,23}$ and an indicator of unfavorable outcome after liver transplantation. ${ }^{24}$

\section{CAUSES OF MALNUTRITION IN END-STAGE LIVER DISEASE}

The liver is the largest metabolic organ of the human body and plays a prime role in the turnover of carbohy- drates, lipids, proteins, vitamins, and trace minerals; it is also an important part of the immune system. Literally all functional properties of the liver are profoundly impaired in end-stage liver disease (ESLD). Malnutrition in patients with ESLD has numerous causes, some of which relate to the underlying etiology of liver damage while others are universal features of declining liver function irrespective of the type of liver disease. Both primary and secondary factors contribute to poor nutritional status and must be accounted for in the management of these patients. The most relevant causes of malnutrition in patients with ESLD are as follows: 1) Dietary insufficiency: a) anorexia, nausea, vomiting; b) early satiety, taste abnormalities, poor palatability of diets (protein and salt restriction); c) reflux disease (ascites, abnormal gut motility): 2) Malabsorbtion: a) pancreatic insufficiency; b) cholestasis (fat soluble vitamins); c) drug-related diarrhoea (lactulose, antibiotics, diuretics, cholestyramine): 3 ) Metabolic disturbances: a) hypermetabolism during complications (infections, haemorrhage, ascitic decompensation); b) protein catabolism (inflammation, impaired liver synthesis); c) impairment of glucose homeostasis due to hepatic insulin resistance (altered gluconeogenesis, low glycogen stores, impaired glycogenolysis); d) increased lipolysis, enhanced lipid oxidation; e) proinflammatory cytokines (TNF $\alpha$, interleukins, leptin): 4) Iatrogenic: a) investigative procedure-related fasting periods; $b$ ) protein restriction during periods of encephalopathy; c) large volume paracentesis.

Notably, the majority of patients with ESLD have no increased resting energy expenditure (REE). A recent study found a normal energy balance in clinically stable cirrhotic patients with malnutrition as assessed by anthropometry. ${ }^{25}$ Seventy-four consecutive cirrhotic patients and nine healthy controls were investigated using indirect calorimetry adjusted according to the patients' physical activity. Thirty-two patients in the cirrhotic group were classified as severely malnourished, but basal energy expenditure (BEE) was similar in all three groups; the non-protein respiratory quotient was lower in cirrhotics notwithstanding their nutritional status. In addition, no difference in the estimated daily energy expenditure and energy intake was observed among groups.

A major reason for primary malnutrition in patients prior to transplantation is reduced food consumption due to anorexia. ${ }^{26}$ Low calorie intake may also be traced to several other reasons including unpalatable diet composition due to salt and protein restriction, ${ }^{23}$ early satiety because of ascites and portal gastropathy, ${ }^{27}$ and loss of appetite due to upregulated mediators of inflammation and mediators of appetite such as tumor necrosis factoralpha and leptin. ${ }^{28,29}$ In addition, in up to $45 \%$ of cirrhotics coexisting infection with Helicobacter pylori may cause 
dyspepsia and a decreased desire for food. ${ }^{30}$ Significant malnutrition may be the result of maldigestion related to pancreatic or biliary abnormalities such as exocrine pancreatic insufficiency or primary biliary liver disorders, while malabsorbtion can result from applied medications such as lactulose or antibiotics causing diarrhea. ${ }^{31}$

Impaired glucose tolerance due to insulin resistance and established diabetes has an important impact on nutritional status in many cirrhotic patients. Due to impaired glyconeogenesis, the cirrhotic liver fails to store sufficient amounts of glycogen; this results in glyconeogenesis from protein catabolism and lipid oxidation. ${ }^{32}$ Therefore, periods of fasting should be avoided in cirrhotic patients, and frequent meals should be implemented to prevent protein catabolism. In fact, late evening meals and nocturnal glucose supplementation has been shown to improve nitrogen balance in cirrhotic patients. ${ }^{33,34}$

\section{NUTRITIONAL ASSESSMENT}

For assessing nutritional status in patients with ESLD on the transplant waiting list no accepted diagnostic "gold standard" exists; in fact, several surrogate markers of an individual's nutritional status are usually necessary to obtain valid data on the severity and pattern of malnutrition.

One useful, easily applicable, and validated approach is subjective global assessment (SGA). This method integrates a detailed medical and dietary history, body weight and height, coexisting medical conditions, and physical activity to rate patients either "well-nourished", "moderately malnourished", or "severely malnourished". The dietary history is ideally recorded by an experienced dietician. SGA is highly specific (96\%) for the detection of malnutrition in liver transplant candidates, ${ }^{35}$ but it lacks sensitivity in patients with severe alcoholic liver disease. ${ }^{36}$

Easily applicable techniques include anthropometric measurements such as body mass index (BMI), triceps skin fold thickness, and mid-arm muscle circumference (MAMC). Unfortunately, most of the easily applicable methods are confounded by significant fluid retention in cirrhotics with ascites and peripheral edema. Reference values for triceps skin fold thickness and MAMC as simple bedside tests for nutritional assessment are given in Table 1. BMI in particular has been criticized for yielding falsely high values, but correction by subtracting estimated amounts of ascites and other fluid collections may compensate for this disadvantage to some extent. ${ }^{8}$

Biochemical markers of malnutrition include serum albumin concentration and measurements of 24-hour creatinine excretion related to a reference population. While the former obviously varies significantly due to hepatic function, the latter has been suggested as an indirect measure of body muscle mass, as $1 \mathrm{~g}$ of excreted creatinine equals $18.5 \mathrm{~kg}$ of muscle mass. ${ }^{37} \mathrm{~A}$ more sophisticated, but less widely available, examination tool for assessing body composition is bioelectric impedance analysis (BIA). BIA is a precise and noninvasive technique that measures lean body mass and fat stores; however, it also becomes inaccurate when patients retain fluid. Another noninvasive method is dual $\mathrm{x}$-ray absorptiometry (DEXA), which provides exact measurements of total body composition. Again, its accuracy declines in patients with ascites and edema. These shortcomings may be bypassed with more precise approaches such as in vivoneutron activation analysis and isotope dilution techniques ${ }^{38}$ but since application of these methods is time-consuming and costly, their use is restricted to research purposes.

Considering these feasibility issues, the European Society for Parenteral and Enteral Nutrition (ESPEN) has published updated guidelines on enteral nutrition in liver transplant candidates. ${ }^{39}$ The current guidelines recommend simple bedside methods such as SGA and/or anthropometry parameters to identify patients at risk for poor nutritional status and BIA to quantify undernutrition despite the limitations of all techniques in patients with ascitic decompensation..$^{39}$ According to the ESPEN expert panel, other composite nutrition scores provide no additional prognostic information.

\section{NUTRITIONAL INTERVENTION - THERAPEUTIC AIMS}

Patients with ESLD on the transplant waiting list frequently display a gradual decline of their nutritional

Table 1 Bedside tests for simple assessment of malnutrition.

\begin{tabular}{lccc}
\hline Anthropometric test & Normal & Moderate & Severe \\
\hline Triceps skin fold thickness & & & \\
Men & $7.5-12.5 \mathrm{~mm}$ & $4-6 \mathrm{~mm}$ & $<4 \mathrm{~mm}$ \\
Women & $10-16.5 \mathrm{~mm}$ & $5-8 \mathrm{~mm}$ & $<5 \mathrm{~mm}$ \\
Mid-arm muscle circumference & $23.0-25.5 \mathrm{~cm}$ & $18-20 \mathrm{~cm}$ & $<18 \mathrm{~cm}$ \\
Men & $21-23 \mathrm{~cm}$ & $6-18.5 \mathrm{~cm}$ & $<16 \mathrm{~cm}$ \\
$\quad$ Women &
\end{tabular}


Table 2 Harris-Benedict equation.

\begin{tabular}{lc}
\hline Gender & Resting energy expenditure \\
\hline Female & $66.5+(9.56 \times$ body weight $[\mathrm{kilogram}])+$ \\
& $(1.85 \times$ height $[$ centimetres] $)-$ \\
& $4.676 \times$ age (years $)$ \\
Male & $66.5+(13.75 \times$ body weight $[$ kilogram] $)+$ \\
& $(5.0 \times$ height [centimetres] $)-$ \\
& $6.75 \times$ age (years) \\
\hline
\end{tabular}

condition. As a result, the major goals of pre-transplant nutritional therapy are to prevent further nutrient and protein depletion and to correct macro- and micronutrient deficiencies. Nutritional support should include the administration of sufficient amounts of calories, proteins, vitamins, minerals, and trace elements without exacerbating liver disease-related complications such as portosystemic encephalopathy, fluid retention, and electrolyte imbalances. Determining the extent of nutritional supplementation requires calculation of the individual's energy needs; this can be done by calculating BEE using the Harris Benedict equation while considering the ideal body weight rather than the patient's actual weight (Table 2). As a rule of thumb, the total calories should be a minimum of 1.2 times the BEE, equalling $35-40 \mathrm{kcal} / \mathrm{kg}$ body weight daily, and $60-70 \%$ should derive from carbohydrates. ${ }^{40}$

Portosystemic encephalopathy is frequent in OLT candidates with ESLD, and many clinicians implement protein restriction to treat it. However, this should be avoided as a routine measure since it aggravates PEM. Instead, encephalopathy should be treated aggressively with standard therapy using lactulose and treatment of precipitating causes such as infections and gastrointestinal hemorrhage. Usually, standard amino acid formulas are well tolerated and should provide at least $1 \mathrm{~g}$ protein/kg body weight per day, which can be increased to $1.2-2.0 \mathrm{~g} / \mathrm{kg}$ daily when tolerated. ${ }^{23,40,41}$ The usefulness of branched-chain amino acids (BCAA) has not been specifically investigated in patients with ESLD on the transplant waiting list, but it can be assumed that the supportive evidence from two recent randomized trials suggesting that long-term ( $<12$ months) nutritional supplementation with oral BCAA is beneficial in slowing the progression of hepatic failure and prolonging eventfree survival in liver cirrhotics also applies for OLT candidates. ${ }^{42,43}$ In practice, whole-protein formulas are generally recommended, and BCAA-enriched formulas should be used in patients who develop encephalopathy during refeeding.

Osteopenia and osteoporosis is frequent in patients with ESLD; therefore, calcium and vitamin D supplementation is recommended for all patients on the waiting list. In those with established osteoporosis or a history of frac- tures, calcium and vitamin D supplementation should be combined with bisphosphonates. ${ }^{23}$

\section{ROUTE OF NUTRITIONAL SUPPORT}

Nutritional supplements should, ideally, be administered enterally, either by oral supplements or, if active eating is hampered, through a gastric or jejunal tube since patients appear to benefit from topical nutritional factors in the gut. Another argument favoring oral nutrition is the lower rate of infections that may occur with central venous catheters. Concerns such as precipitating variceal hemorrhage while inserting the feeding tube have not been confirmed in clinical trials. ${ }^{44}$ However, reports of complications related to malpositioned feeding tubes continue to surface; most are due to inadvertent dislocation in the respiratory tract causing aspiration, especially when the tube is placed in the esophagus. Other complications observed occasionally with nasogastric/ nasoduodenal tubes include epistaxis, sinusitis, tube removal or retraction, tube clogging, and tube-feedingassociated diarrhea. ${ }^{45,46}$ However, complications related to malpositioned feeding tubes are usually preventable if correct placement is safely achieved and regularly monitored.

Total parenteral nutrition (TPN) should be restricted to patients who are unable to eat or those for whom enteral feeding is contraindicated. In cases of severe gastrointestinal dysfunction, such as esophageal bleeding or intestinal obstruction, TPN remains an option to ensure adequate caloric intake. However, TPN is associated with higher risks of infection and electrolyte imbalance, it is more expensive, and since evidence supporting its use in ESLD stems from studies focused on the treatment of severe alcoholic liver disease, it may not apply to patients waiting for liver transplantation. ${ }^{9,23}$

\section{NUTRITIONAL THERAPY BEFORE LIVER TRANSPLANTATION}

Until now, only two prospective controlled trials investigated the effect of pre-transplant nutritional therapy on the outcome of patients undergoing OLT. Chin et al. ${ }^{47}$ prospectively included 19 children with ESLD, with a median age of 1.25 years, to compare a high-energy, isoenergetic and isonitrogenous BCAA-enriched semielemental formulation with a matched standard semielemental formation. Only 12 of 19 patients completed the study before OLT, and only 10 of 19 completed a full crossover study. Both regimens improved weight and height, whereas the BCAA formula resulted in significantly more pronounced improvements of total body potassium, mid-upper-arm circumference, and subscapu- 
lar skin fold thickness in comparison to the standard formulation. Also, significantly fewer albumin infusions were required during the BCAA supplement. The authors concluded that BCAA-enriched formulas seem to have advantages over standard semi-elemental formulas for improving nutritional status in children with ESLD listed for OLT. Obviously, the low number of patients limits the significance of these results. A slightly larger prospective randomized controlled trial from the United Kingdom examined the effect of pre-transplant nutritional supplementation on the outcome of 82 adult patients (42 with active enteral nutritional therapy in addition to regular diet; 40 control patients without additional nutrition) undergoing liver transplantation. ${ }^{48}$ Nutritional supplementation improved mid-arm circumference, mid-arm muscle circumference, and grip strength. However, supplementation did not affect survival, although there were more deaths in the control group than there were in the supplemented group (nine versus five, $p=0.075$ ). Although the importance of nutritional therapy for patients with ESLD listed for OLT is generally accepted, ${ }^{49}$ clear scientific evidence supporting this view is scarce and no study has demonstrated that preoperative nutritional therapy of whatever kind improves the outcome of liver transplantation.

\section{NUTRITIONAL THERAPY AFTER LIVER TRANSPLANTATION}

Although most nutritional deficiencies and metabolic disturbances prevalent in patients with ESLD rapidly improve following successful OLT, preoperative malnutrition, surgical stress, post-interventional complications, postoperative protein catabolism, and fasting periods suggest the need for early nutritional support after OLT. Expert guidelines recommend that 1.5$2.0 \mathrm{~g}$ protein $/ \mathrm{kg}$ body weight should be administered daily to liver transplant recipients during the immediate post-transplant period; this is to compensate for the significant loss of nitrogen caused by the excretion of large amounts of urinary nitrogen. ${ }^{23,40}$ Ideally, nutrition should be given either orally or enterally via a nasogastric tube since this is associated with lower postoperative rates of infection ${ }^{50}$ and fewer metabolic disturbances ${ }^{51}$ than TPN.

So far, only a few clinical studies have elucidated the role of postoperative nutritional therapy in patients who received a liver transplant. Reilly et al..$^{52}$ investigated a total of 28 patients for the effects of two TPN regimens, either with standard amino acids or BCAA immediately after OLT, in comparison with the effects of a glucose infusion administered for 7 days. Both TPN treatment regimens were equally effective and performed better than the glucose infusion, as reflected by an improved nitrogen balance and a shorter duration of ventilation therapy and stay on the intensive care unit. This small study provided supportive evidence for nutritional therapy in general but clearly neglected possible benefits from oral or enteral feeding. The latter issue was addressed in another study that randomized 50 transplant patients to receive either an enteral formula via nasointestinal feeding tubes or maintenance intravenous fluid until oral diets were initiated..$^{51}$ Only 31 patients completed the trial for evaluation. The tube-fed patients $(\mathrm{N}=14)$ showed significantly higher total nutrient intakes, a better nitrogen balance, and developed infections less frequently. Early post-transplant tube feeding did not influence hospitalization costs, hours on the ventilator, lengths of stay in the intensive care unit and hospital, or rejection during the first 21 days post-transplant.

Only one study directly compared early parenteral with enteral nutrition in the immediate post-operative phase after OLT. ${ }^{53}$ Patients were randomly assigned 28 hypoalbuminemic cirrhotic patients immediately after liver transplantation to receive either no nutritional support $(\mathrm{N}=10)$, TPN providing $35 \mathrm{kcal} / \mathrm{kg} /$ day with standard amino acids at $1.5 \mathrm{~g} / \mathrm{kg} /$ day $(\mathrm{N}=8)$, or isocaloric isonitrogenous TPN with added BCAA $(\mathrm{N}=10)$ for 7 days post-transplant. Both TPN groups showed a better nitrogen balance, and a (non-significant) shorter time on ventilation and faster discharge from the intensive care unit.

To conclude, early post-transplant nutritional therapy seems to improve a number of surrogates of nutritional status in recipients, but clear evidence for an improvement of post-transplant outcome is lacking.

An innovative approach was chosen in a recent pilot study from New Zealand, which investigated the therapeutic benefit from pre- and post-operative immunonutrition in liver transplant patients. ${ }^{54}$ The underlying rationale for using immuno-nutrition is based on the observation that post-operative nutritional supplementation with a formula consisting of arginine, fish oil, and nucleotides proved to be beneficial for reducing post-operative infectious complications in patients undergoing gastrointestinal surgery; this is possibly due to down-regulatory effects on inflammatory responses and amelioration of postoperative immunosuppression rather than due to effects on nutrition per se. ${ }^{55}$ Fifteen consecutive patients received a commercial immuno-nutritional preparation $(0.6 \mathrm{~L} / \mathrm{d})$ for a median of 54 days pre- and post-transplant in a non-randomized fashion; outcome was compared to that of 17 patients who received standard nutritional intervention. Immuno-nutritional intervention was tolerated very well and resulted in non-significant improvements of pre-operative nutritional status, 
accelerated recovery after transplantation, and reduced postoperative infectious complications. Although the study was small and uncontrolled, the favorable results seem to justify a subsequent controlled trial.

Rayes et al..$^{56}$ carried out a prospective randomized double-blind trial in 66 liver transplant recipients who received enteral nutrition in the immediate pre- and post-operative periods. Outcome was compared between a group receiving a commercial composition of four strains of lactic acid bacteria and four fibers and another group receiving the fibers only. Treatment started the day before surgery and continued for 14 days. The incidence of post-operative bacterial infections was $48 \%$ in the group receiving fibers only and $3 \%$ in those receiving $\mathrm{LAB}$ and fibers $(P<0.05)$. In addition, the duration of antibiotic therapy was significantly shorter in the latter group. In both groups, only mild or moderate infections occurred. Although this study did not choose nutritional endpoints to assess therapeutic effects, the results suggest a possible benefit from certain "neutriceutic" on important determinants of post-operative outcome.

ESPEN has recently issued a detailed statement on guidelines regarding the management of patients with ESLD, liver transplant candidates, and subjects needing major surgical interventions. ${ }^{39}$ Herein, recommendations regarding patients awaiting liver transplantation largely overlap with those issued for patients with liver cirrhosis. Details of these recommendations are summarized in Table 3.

\section{CONCLUSIONS}

Nutritional aspects are important both for patients awaiting liver transplantation and for those who live on with a liver graft. While pre-transplant patients and those in the immediate post-transplant period usually require correction of numerous nutritional deficiencies to improve their overall condition, post-transplant liver patients have to be followed up to ensure they do not develop over nutrition. Both settings require an interdisciplinary approach that integrates the expertise of physicians, surgeons, nutritionists, and nursing staff who are well-aware of these issues. Only with joint efforts can nutritional imbalances be overcome to optimize the outcome of patients in liver transplant programs.

\section{Acknowledgment}

FS is supported by research grants from the Novartis Foundation for Scientific Research.

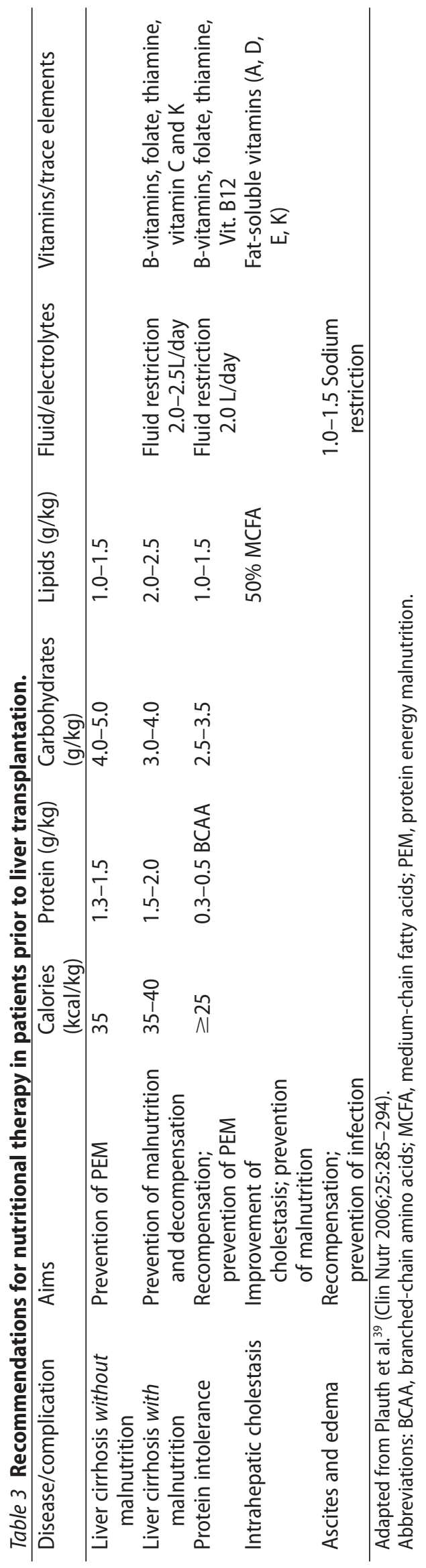

Nutrition Reviews ${ }^{\circledast}$ Vol. 66(1):47-54 


\section{REFERENCES}

1. Child CG, Turcotte JG. Surgery and portal hypertension. In: The Liver and Portal Hypertension. Child CG (ed). Philadelphia: Saunders; 1964:50-64.

2. Kamath PS, Kim WR; Advanced Liver Disease Study Group. The model for end-stage liver disease (MELD). Hepatology 2007;45:797-805.

3. McCullough AJ, Bugianesi E. Protein calorie malnutrition and the etiology of cirrhosis. Am J Gastroenterol 1997;92:734738.

4. Merli M, Riggio O, Dally L. Does malnutrition affect survival in cirrhosis? Hepatology 1996;23:1041-1046.

5. Müller MJ, Lautz HU, Plogmann B, Burger M, Korber J, Schmidt FW. Energy expenditure and substrate oxidation in patients with cirrhosis: the impact of cause, clinical staging and nutritional state. Hepatology 1992;15:782-794.

6. Italian Multicentre Cooperative Project on Nutrition in Liver Cirrhosis. Nutritional status in cirrhosis. J Hepatol 1994; 21:317-325.

7. Caregaro L, Alberino F, Amodio P, et al. Malnutrition in alcoholic and virus-related cirrhosis. Am J Clin Nutr 1996;63:602609.

8. Figueiredo FA, Dickson ER, Pasha TM, et al. Utility of standard nutritional parameters in detecting body cell mass depletion in patients with end-stage liver disease. Liver Transpl 2000; 6:575-5781.

9. Stickel F, Hoehn B, Schuppan D, Seitz HK. Nutritional therapy in alcoholic liver disease. Aliment Pharmacol Ther 2003; 18:357-373.

10. Lochs H, Plauth M. Liver cirrhosis: rationale and modalities for nutritional support - the European Society of Parenteral and Enteral Nutrition consensus and beyond. Curr Opin Clin Nutr Metab Care 1999;2:345-349.

11. McCormick PA, Morgan MY, Phillips A, et al. The effects of alcohol use on rebleeding and mortality in patients with alcoholic cirrhosis following variceal hemorrhage. J Hepatol 1992;14:99-103.

12. Sanchez AJ, Aranda-Michel J. Nutrition for the liver transplant patient. Liver Transpl 2006;12:1310-1316.

13. Merli M, Nicolini G, Angeloni S, Riggio O. Malnutrition is a risk factor in cirrhotic patients undergoing surgery. Nutrition 2002;18:978-986.

14. Kondrup J. Nutrition in end stage liver disease. Best Pract Res Clin Gastroenterol 2006;20:547-560.

15. Gloria L, Cravo M, Camilo ME, et al. Nutritional deficiencies in chronic alcoholics: relation to dietary intake and alcohol consumption. Am J Gastro 1997;92:485-489.

16. Levy S, Herve C, Delacoux E, Erlinger S. Thiamine deficiency in hepatitis $C$ virus and alcohol-related liver diseases. Dig Dis Sci 2002;47:543-548.

17. Leo MA, Lieber CS. Hepatic vitamin A depletion in alcoholic liver injury. N Engl J Med 1982;307:597-601.

18. Leo MA, Lieber CS. Alcohol, vitamin A, and beta-carotene: adverse interactions including hepatotoxicity and carcinogenensis. Am J Clin Nutr 1999;69:1071-1085.

19. Dan Z, Popov Y, Patsenker E, et al. Hepatotoxicity of alcoholrelated polar retinoid metabolites involves apoptosis via loss of mitochondrial membrane potential. FASEB J 2005;19:845847.

20. Marchesini G, Fabbri A, Bianchi G, Brizi M, Zoli M. Zinc supplementation and amino acid-nitrogen metabolism in patients with advanced cirrhosis. Hepatology 1996;23:10841092.
21. Bresci G, Parisi G, Banti S. Management of hepatic encephalopathy with oral zinc supplementation: a long-term treatment. Eur J Med 1993;2:414-416.

22. Selberg O, Bottcher J, Tusch G, Pichlmayr R, Henkel E, Muller MJ. Identification of high- and low-risk patients before liver transplantation: a prospective cohort study of nutritional and metabolic parameters in 150 patients. Hepatology 1997;25:652-657.

23. Figueiredo F, Dickson ER, Pasha T, et al. Impact of nutritional status on outcomes after liver transplantation. Transplantation 2000;70:1347-1352.

24. Aranda-Michel J. Nutrition in hepatic failure and liver transplantation. Curr Gastroenterol Rep 2001;3:362-370.

25. Riggio O, Angeloni S, Ciuffa L, et al. Malnutrition is not related to alterations in energy balance in patients with stable liver cirrhosis. Clin Nutr 2003;22:553-559.

26. Davidson HI, Richardson RA, Sutherland D, Garden OJ. Macronutrient preference, dietary intake, and substrate oxidation among stable cirrhotic patients. Hepatology 1999;29:13801386.

27. Aqel BA, Scolapio JS, Dickson RC, Burton DD, Bouras EP. Contribution of ascites to impaired gastric function and nutritional intake in patients with cirrhosis and ascites. Clin Gastroenterol Hepatol 2005;3:1095-1100.

28. Langhans W. Role of the liver in the metabolic control of eating: what we know - and what we do not know. Neurosci Biobeh Rev 1996;20:145-153.

29. McCullough AJ, Bugianesi E, Marchesini G, et al. Genderdependent alterations in serum leptin in alcoholic cirrhosis. Gastroenterology 1998;115:947-953.

30. Kamalaporn P, Sobhonslidsuk A, Jatchavala J, et al. Factors predisposing to peptic ulcer disease in asymptomatic cirrhotic patients. Aliment Pharmacol Ther 2005;21:14591465.

31. Merli M, Caschera M, Piat C, Pinto G, Diofebi M, Riggio O. The effect of lactulose and lactitol administration on fecal fat excretion in patients with liver cirrhosis. J Clin Gastroenterol 1992;15:125-127.

32. Riggio O, Merli M, Leonetti $F$, et al. Impaired nonoxidative glucose metabolism in patients with liver cirrhosis: effects of two insulin doses. Metabolism 1997;46:840-843.

33. Swart GR, Zillikens MC, van Vuure JK, Van den Berg JW. Effect of a late evening meal on nitrogen balance in patients with cirrhosis of the liver. Brit Med J 1989;299:1202-1203.

34. Zillikens $M C$, van den Berg JW, Wattimena JL, Rietveld $T$, Swart GR. Nocturnal oral glucose supplementation. The effects on protein metabolism in cirrhotic patients and in healthy controls. J Hepatol 1993;17:377-383.

35. Hasse J, Strong S, Gorman MA, Liepa G. Subjective global assessment: alternative nutrition-assessment technique for liver-transplant candidates. Nutrition 1993;9:339-343.

36. Naveau S, Belda E, Borotto E, Genuist F, Chaput JC. Comparison of clinical judgment and anthropometric parameters for evaluating nutritional status in patients with alcoholic liver disease. J Hepatol 1995;23:234-235.

37. Pirlich M, Selberg O, Boker K, et al. The creatinine approach to estimate skeletal muscle mass in patients with cirrhosis. Hepatology 1996;24:1422-1427.

38. Nielsen K, Kondrup J, Martinsen L, et al. Nutritional assessment and adequacy of dietary intake in hospitalized patients with alcoholic liver cirrhosis. Br J Nutr 1993;69:665679.

39. Plauth $M$, Cabre E, Riggio 0 , et al. ESPEN guidelines on enteral nutrition: liver disease. Clin Nutr 2006;25:285-294. 
40. Campos AC, Matias JE, Coelho JC. Nutritional aspects of liver transplantation. Curr Opin Clin Nutr Metab Care 2002;5:297307.

41. Harrison J, McKiernan J, Neuberger J. A prospective study on the effect of recipient nutritional status on outcome in liver transplantation. Transplant Int 1997;10:369-374.

42. Marchesini G, Bianchi G, Merli M, et al. Nutritional supplementation with branched-chain amino acids in advanced cirrhosis: a double-blind, randomized trial. Gastroenterology 2003;124:1792-1801.

43. Muto $Y$, Sato $S$, Watanabe A, et al. Effects of oral branchedchain amino acid granules on event-free survival in patients with liver cirrhosis. Clin Gastroenterol Hepatol 2005;3:705713.

44. Calvey H, Davis M, Williams R. Controlled trial of nutritional supplementation, with and without branched chain amino acids enrichment, in treatment of acute alcoholic hepatitis. J Hepatol 1985;1:141-151.

45. Metheny NA, Meert KL, Clouse RE. Complications related to feeding tube placement. Curr Opin Gastroenterol 2007; 23:178-182.

46. Baskin WN. Acute complications associated with bedside placement of feeding tubes. Nutr Clin Pract 2006;21:40-55.

47. Chin SE, Shepherd RW, Thomas BJ, et al. Nutritional support in children with end-stage liver disease: a randomized crossover trial of a branched-chain amino acid supplement. Am J Clin Nutr 1992;56:158-163.

48. Le Cornu KA, McKiernan FJ, Kapadia SA, Neuberger JM. A prospective randomized study of preoperative nutritional supplementation in patients awaiting elective orthotopic liver transplantation. Transplantation 2000;69:1364-1369.
49. Henkel AS, Buchman AL. Nutritional support in patients with chronic liver disease. Nat Clin Pract Gastroenterol Hepatol 2006;3:202-209.

50. Moore FA, Feliciano DV, Andrassy RJ, et al. Early enteral feeding, compared with parenteral, reduces postoperative septic complications. The results of a meta-analysis. Ann Surg 1992;216:172-183.

51. Hasse JM, Blue LS, Liepa GU, et al. Early enteral nutrition support in patients undergoing liver transplantation. J Parenter Enteral Nutr 1995;19:437-443.

52. Reilly J, Mehta R, Teperman L, et al. Nutritional support after liver transplantation: a randomized prospective study. J Parenter Enteral Nutr 1990;14:386-391.

53. Wicks C, Somasundaram S, Bjarnason I, et al. Comparison of enteral feeding and total parenteral nutrition after liver transplantation. Lancet 1994;344:837-840.

54. Plank LD, McCall JL, Gane EJ, et al. Pre- and postoperative immunonutrition in patients undergoing liver transplantation: a pilot study of safety and efficacy. Clin Nutr 2005; 24:288-296.

55. Senkal M, Zumtobel V, Bauer KH, et al. Outcome and costeffectiveness of perioperative enteral immunonutrition in patients undergoing elective upper gastrointestinal tract surgery: a prospective randomized study. Arch Surg 1999; 134:1309-1316.

56. Rayes N, Seehofer D, Theruvath T, et al. Supply of pre- and probiotics reduces bacterial infection rates after liver transplantation - a randomized, double-blind trial. Am J Transplant 2005;5:125-130. 